\title{
Correction to: Archeological neuroimmunology: resurrection of a pathogenic immune response from a historical case sheds light on human autoimmune encephalomyelitis and multiple sclerosis
}

\author{
Eduardo Beltrán ${ }^{1} \cdot$ Manuela Paunovic $^{2} \cdot$ David Gebert $^{1} \cdot$ Emine Cesur $^{2} \cdot$ Markus Jeitler $^{3} \cdot$ Romana Höftberger $^{4}$. \\ Joachim Malotka ${ }^{1}$. Simone Mader ${ }^{1} \cdot$ Naoto Kawakami $^{1} \cdot$ Edgar Meinl $^{1} \cdot$ Monika Bradl $^{2} \cdot$ Klaus Dornmair $^{1,5}$. \\ Hans Lassmann² ${ }^{2}$
}

Published online: 9 February 2021

c) Springer-Verlag GmbH Germany, part of Springer Nature 2021

\section{Correction to: Acta Neuropathologica (2021) 141:67-83 https://doi.org/10.1007/s00401-020-02239-2}

Due to an error in the final editing of our manuscript the injection volume of intrathecal antibody application (page 72) was stated as $100 \mathrm{ml}$ instead of 100 microliter.

Publisher's Note Springer Nature remains neutral with regard to jurisdictional claims in published maps and institutional affiliations.

The original article can be found online at https://doi.org/10.1007/ s00401-020-02239-2.

Hans Lassmann

hans.lassmann@meduniwien.ac.a

1 Institute of Clinical Neuroimmunology, University Hospital and Biomedical Center, Ludwig-Maximilians University Munich, Munich, Germany

2 Department of Neuroimmunology, Center for Brain Research, Medical University of Vienna, Spitalgasse 4, 1090 Vienna, Austria

3 Core Facility Genomics, Medical University Vienna, Vienna, Austria

4 Division of Neuropathology and Neurochemistry, Department of Neurology, Medical University of Vienna, Vienna, Austria

5 Munich Cluster for Systems Neurology (SyNergy), Munich, Germany 